\title{
Memory for objects in canonical and noncanonical viewpoints
}

\author{
Pablo Gomez and Jennifer Shutter \\ DePaul University, Chicago, Illinois \\ AND \\ JEFFREY N. ROUDER \\ University of Missouri, Columbia, Missouri
}

\begin{abstract}
This article investigates how the perspective from which we see an object affects memory. Object identification can be affected by the orientation of the object. Palmer, Rosch, and Chase (1981) coined the term canonical to describe perspectives in which identification performance is best. We present two experiments that tested the effects of object perspective on memory. Our results revealed a double dissociation between task (recognition and recall) and type of object perspective. In recognition, items studied in the noncanonical viewpoint produced higher proportions of "old" responses than did items studied in the canonical viewpoint, whereas new objects presented from a noncanonical viewpoint produced fewer "old" responses than did new objects presented from the canonical viewpoint. In free recall, conversely, objects studied from the noncanonical viewpoint produced lower recall rates than did objects studied from the canonical viewpoint. These results, which reveal a pattern similar to word frequency effects, support the psychological reality of canonical viewpoints and the frequencyof-exposure-based accounts of canonical viewpoint effects.
\end{abstract}

The present article is concerned with a simple question: Does the viewpoint from which we see a 3-D object affect our memory for that object? The consideration of the role of viewpoint in object recognition has an involved history. It has been shown repeatedly that viewpoint affects identification performance. Palmer, Rosch, and Chase (1981) found the following viewpoint effects: (1) Viewpoint affected ratings of goodness of photographs of familiar objects; (2) viewpoint affected the formation and description of a mental image of a familiar object; and (3) viewpoint affected response times and error rates in naming objects. Moreover, the viewpoint that maximized object-identification performance was often considered by participants to be the best; it was also the easiest for participants to imagine and describe. Palmer et al. coined the term canonical to describe this seemingly best view. Additional evidence for the existence of canonical viewpoints was provided by Blanz, Tarr, and Bülthoff (1999), Jolicœur (1985), and Verfaillie and Boutsen (1995).

The role of viewpoint in object recognition has been debated vigorously. Several theorists have cited viewpoint effects as evidence that viewpoint-specific representations underlie object recognition (Edelman \& Bülthoff, 1992; Tarr \& Bülthoff, 1995). Others have posited that objects are stored in a 3-D representation without specific, privileged viewpoints (Biederman, 1987). Canonical viewpoint effects do occur; hence, for the latter theories to hold, the effects must reflect decision variables rather than result from matches or mismatches to underlying representations (see, e.g., Rouder, Ratcliff, \& McKoon, 2000).

One explanation of canonical viewpoints that is consistent with both viewpoint-specific and viewpoint-irrelevant theories is that they represent a frequency effect. Tarr and Pinker (1989), for example, suggested that canonical viewpoints for novel objects form only after repeated viewings at a specific orientation. Karnath, Ferber, and Bülthoff (2000) suggested that the neurons that underlie the recognition of an object become attuned to the features present in the most recognizable views of that object, and that this tuning evolves with experience.

The notion that frequency affects memory is not restricted to the effects on object recognition. Frequency of occurrence is one of the most studied variables in the memory for words. The effect of frequency of occurrence is surprisingly complex and varies with the memory task. In recognition memory paradigms, in which participants are asked to judge whether words at test were previously studied, there is an advantage for low-frequency words over high-frequency words (Jacoby \& Dallas, 1981; Peters, 1936; Scarborough, Cortese, \& Scarborough, 1977; Underwood \& Freund, 1970). Moreover, this advantage often exhibits the mirror effect (Hockley, 1994): Studied low-frequency words are more often identified as old than are studied high-frequency words, and new low-frequency

P. Gomez, pgomez1@depaul.edu 
words are more often identified as new than are new highfrequency words (Glanzer \& Bowles, 1976). The case for recall is the opposite: High-frequency words hold a recall advantage over low-frequency words in cued recall, free recall (in particular, in blocked-by-frequency lists; e.g., Gillund \& Shiffrin, 1984), and serial recall (Hall, 1954; Shepard, 1967; Underwood, Ekstrand, \& Keppel, 1965).

In the present article, we consider the hypothesis that viewpoint plays a role analogous to word frequency, with canonical viewpoints mapping to high-frequency words and noncanonical viewpoints mapping to low-frequency words. This hypothesis, which we call the viewpoint-asfrequency hypothesis, predicts better memory performance in a recall task for canonical viewpoints than for noncanonical viewpoints. It predicts the reverse for recognition tasks, however: Better memory performance will result from noncanonical viewpoints than from canonical viewpoints.

There are alternatives to the viewpoint-as-frequency hypothesis. For example, differences in viewpoint may lead to variations in the extent to which items are processed; hence, orientation might affect the strength of the memory trace. Noncanonical viewpoints might induce participants to perform extra processing (e.g., mental rotation), which might yield differential attention. In this case, items studied using more attentional resources would be both better recognized and better recalled. Yet another possibility is that objects presented from canonical viewpoints are studied at a deeper level than are those presented from noncanonical viewpoints. This increased depth of processing might lead a canonical viewpoint to produce a stronger memory trace, and thus to produce an advantage in both recognition and recall.

\section{Overview of the Experiments}

We report two experiments designed to test the differing predictions of the viewpoint-as-frequency hypothesis and the viewpoint-as-strength hypothesis. In Experiment 1, participants performed a recognition memory task in which viewpoint was manipulated both at study and at test, and these conditions were factorially combined. The two combinations most useful for assessing the hypothesis were (1) when the object was both studied and tested in the canonical orientation, and (2) when the object was both studied and tested in the noncanonical orientation. These two conditions are matched conditions. The remaining two conditions are mismatched conditions, in which orientation changed across study and test. The latter two conditions were included for completeness, since this study is one of the first to examine orientation across memory tasks. In retrospect, however, these mismatched conditions, which cannot be described as either canonical or noncanonical, have little bearing on the hypothesis. There were also two different testing instructions: In the viewpoint-irrelevant condition, test items were to be considered "old" regardless of their orientation during study; in the viewpoint-relevant condition, test items were to be considered "old" only if they were presented in the same orientation in the study phase as in the test phase. The hypotheses above apply equally to these testing conditions; hence, finding the same effect would provide for generality of the findings. In Experiment 2, participants performed a free-recall task in which viewpoint was manipulated at study.

\section{EXPERIMENT 1}

\section{Method}

Participants. Forty-two DePaul University undergraduate students served as participants for course credit in an introductory psychology course

Materials. Experimental stimuli consisted of 342 images of 171 common objects. The images consisted of one canonical view and one noncanonical view of each of the 171 objects. The images were developed using the 3D Studio MAX, Version 7 graphics software package and free 3-D models available at www.3dcafe.com, www.amazing3d .com, and ftp://ftp.uniovi.es/pub/mirrors/avalon.viewpoint.com.

The 171 familiar objects were selected to be similar to Palmer et al.'s (1981) stimuli. When several instances of the same object were available (e.g., different cars), only one was used. Perspectives of the objects were manipulated using the graphics software. Rotations of the 3-D models along their vertical and/or horizontal axis into canonical and noncanonical perspectives were rendered into 2-D images in a neutral, light gray background (see Figure 1 for an example). The amount of rotation of the model was manipulated to achieve canonical and noncanonical perspectives. Canonical perspectives were based on the most familiar perspective of an object. Noncanonical perspectives were based on the least familiar perspective of an object that nevertheless allowed it to remain recognizable. Six graduate students were presented with initial versions of the canonical and noncanonical viewpoints. On the basis of their recommendations, the images were maintained, modified, or removed. If more than one member of the panel disagreed with the canonical perspective presented, the item was removed. In addition, a full-color survey instrument depicting objects representing the noncanonical perspectives of roughly 180 objects was created and was administered to 28 introductory psychology undergraduate students. The survey instrument was designed to ensure that the objects in the images representing the noncanonical perspectives were recognizable. If more than $10 \%$ of the raters were unable to identify an object, the noncanonical image was altered or discarded. Images that had been discarded because of disagreement regarding their canonical/noncanonical perspective served as buffers in the study lists.

Design. Object orientation (canonical or noncanonical) was manipulated at study and at test, yielding four combinations. Additionally, the task instructions were manipulated through two levels. In the viewpoint-relevant condition, participants were instructed to consider a test object as "old" only if it was studied in the same orientation, and as "new" in any other case. In the viewpointirrelevant condition, participants were instructed to consider a test
A

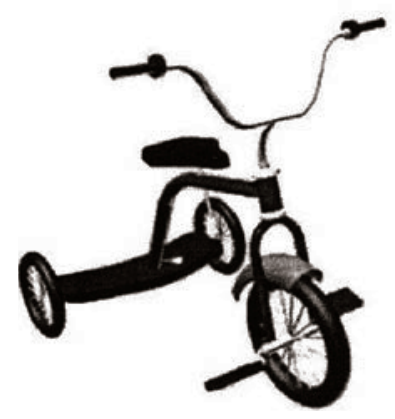

B

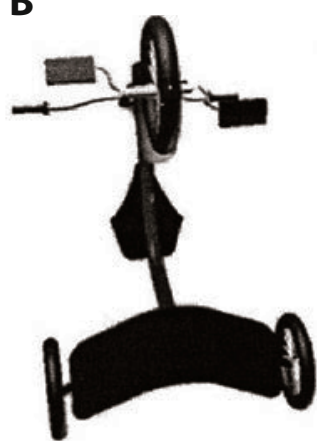

Figure 1. A tricycle from the canonical (A) and noncanonical (B) viewpoints. 
object as "old" if it was studied at any orientation. Orientation at test and study was manipulated within blocks of items. Instruction conditions were manipulated within participants but across blocks. Half of the participants performed under the viewpointirrelevant condition for the first half of the blocks, and under the viewpoint-relevant condition for the second half. The other half of the participants followed the reverse order. Viewpoints were counterbalanced across participants, as was the order of viewpointrelevancy instructions.

Procedure. Experiment 1 consisted of 14 blocks of trials that were subdivided into study and test phases. During the study phase, equal numbers of canonical and noncanonical views were presented. There were 16 critical objects. Additionally, 3 images (of objects for which raters could not agree on canonical/noncanonical viewpoints) preceded the 16 critical objects and served as a primacy buffer; likewise, 3 images followed the 16 critical objects and served as a recency buffer. The primacy and recency buffers were not considered in the subsequent analysis. During the test phase, equal numbers of canonical and noncanonical views were presented. Moreover, all four study-test orientation combinations were presented equally often within each block. In addition, 8 new test objects were interspersed throughout the list. Participants pressed the "?" or " $Z$ " key on a computer keyboard to indicate whether a tested item was studied or novel, respectively. Participants saw only one version of each object throughout the experiment.

\section{Results}

Data from 6 participants were removed for the following reasons: Two participants produced the same response on all trials; and 4 participants failed to follow the viewpoint-relevancy instructions, as evidenced by an error rate of at least $70 \%$. Task order (viewpoint-relevant first or last) did not produce significant effects $(F<1)$, so we averaged across this variable. Figure 2 provides the proportion of "old" responses for the viewpoint-irrelevant (left) and viewpoint-relevant (center) conditions. As mentioned previously, the viewpoint-as-frequency hypothesis was evaluated for the contrast between objects that were both studied and tested in the canonical view (C) versus those that were both studied and tested in the noncanonical view $(\mathrm{N})$. In Figure 2, solid lines connect the hit rates for these items, and false alarm rates are connected by dotted lines. Performance from conditions that could not unambiguously be considered noncanonical or canonical was plotted (using lowercase letters), but it was not analyzed further. For the viewpoint-irrelevant condition, there was a significant increase in the hit rate for noncanonical items $[p($ hit $)=.848]$ over that for canonical items $[p$ (hit $)=$ $\left..789 ; t(35)=2.59, p=.014, p_{\text {rep }}=.80\right]$, as well as a significant decrease in false alarm rate for new canonical items $[p(\mathrm{FA})=.182]$ versus new noncanonical items $\left[p(\mathrm{FA})=.108 ; t(35)=3.586, p<.001, p_{\text {rep }}=.84\right]$.

The interpretation for the viewpoint-relevant condition was slightly more complex, because the memory advantage for noncanonical items was not as pronounced as it was in the viewpoint-irrelevant condition. Although there was no difference in hit rates $[.782$ vs. $.771 ; t(35)=0.740$,

\section{A Viewpoint-Irrelevant B Viewpoint-Relevant}

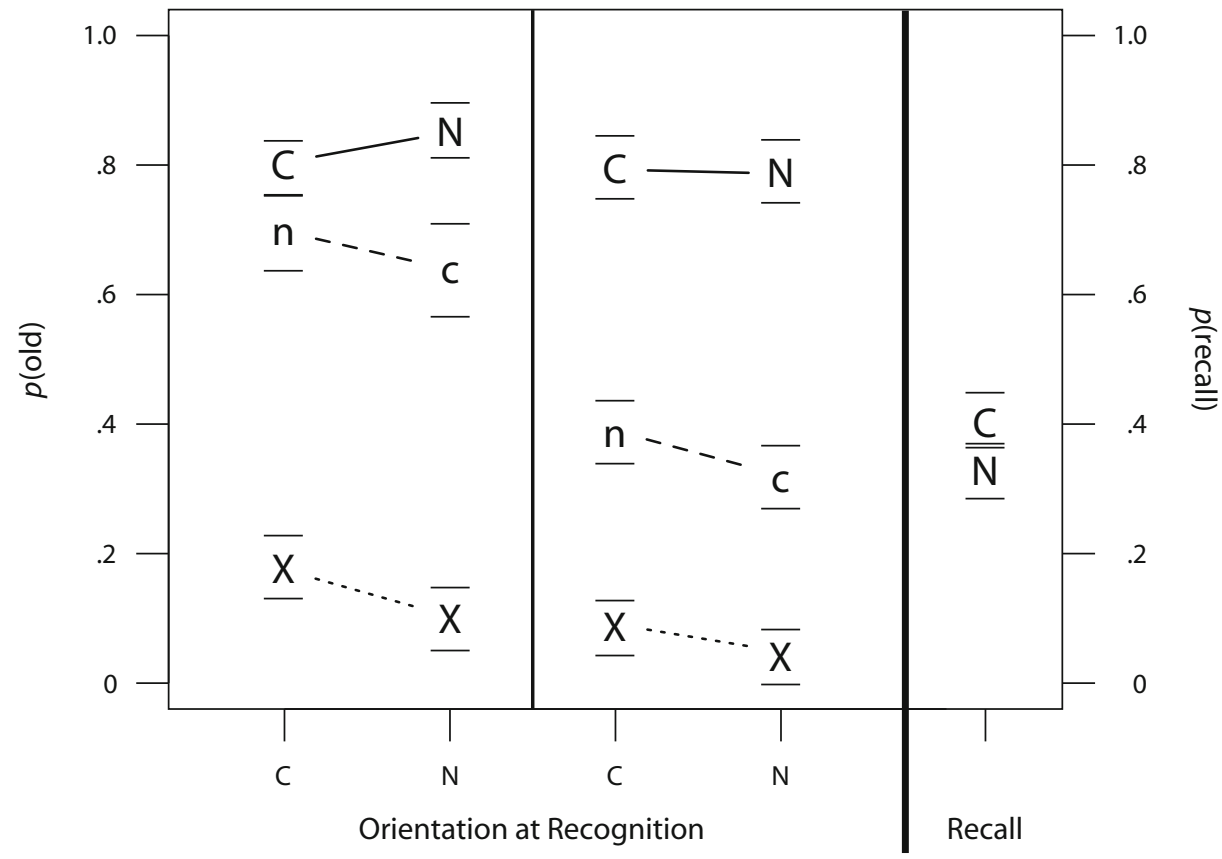

Figure 2. Results from Experiments 1 and 2. Panels $A$ and $B$ show the results from the viewpointirrelevant and viewpoint-relevant conditions in Experiment 1 (recognition memory), respectively. The solid lines denote the conditions in which study and test orientation matched, the dashed lines denote the conditions in which the study and test conditions mismatched, and the dotted lines denote the new items. The $x$-axis indicates the orientation during the test phase in the recognition memory tasks; the letter denotes the orientation of the object during study (C/c, canonical; N/n, noncanonical; $X$, new objects). The horizontal lines above and below the symbols denote $95 \%$ confidence intervals. 
$\left.p=.464, p_{\text {rep }}=.57\right]$, there was an advantage for noncanonical items in false alarm rates [.057 vs. .097; $t=2.933$, $\left.p=.006, p_{\text {rep }}=.78\right]$, indicating an overall mnemonic advantage for noncanonical items.

The results from the two conditions, taken together, indicate that recognition memory was better for noncanonical viewpoints than for canonical viewpoints, with this advantage being reduced in the viewpoint-relevant condition. In the viewpoint-irrelevant condition, the effect of viewpoint displays a mirror effect (Glanzer \& Adams, 1985), much like that observed in the effect of frequency on the memorability of words. In the viewpoint-relevant condition, however, the effect was observed on the false alarm rate only.

\section{EXPERIMENT 2}

\section{Method}

Experiment 2 was a free-recall test. Many aspects of the experiment, including the materials and the blocks of study and test phases, were identical to those of Experiment 1. The differences were as follows.

Participants. Forty-three DePaul undergraduate students served as participants for course credit in an introductory psychology course.

Procedure. In the test phase of Experiment 2, participants were given a blank piece of paper and were asked to recall, by name, all of the items in the study list. There was no time restriction imposed on participants: Responses were self-paced.

\section{Results}

The buffer items were not analyzed and are not reported here. The proportion of recalled items that were studied in the canonical condition (.406) was higher than that of items that were studied in the noncanonical condition (.330); this difference was highly significant $[t(42)=$ $\left.5.658, p<.001, p_{\text {rep }}=.96\right]$.

\section{DISCUSSION}

Our results revealed a crossover interaction between task (recognition and recall) and type of item (canonical and noncanonical). In recognition, items studied in the noncanonical viewpoint produced higher proportions of "old" responses across almost all conditions, whereas new objects tested from a noncanonical viewpoint produced fewer "old" responses than did new objects tested from the canonical viewpoint. In free recall, however, objects studied from the noncanonical viewpoint produced lower recall rates than did objects studied from the canonical viewpoint.

The pattern of viewpoint effects that is presented here is analogous to that of word frequency effects; namely, highfrequency words are recalled better than low-frequency words (although this finding is not very robust in mixed lists like ours), whereas low-frequency words are recognized better than high-frequency words. Karlsen and Snodgrass (2004) reported a similar result with pictures of familiar and nonfamiliar objects in a blocked-byfamiliarity recognition memory experiment. They noted that the verbal labels also varied in frequency. We avoided this issue, because the labels were the same regardless of the viewpoint. This might explain our ability to find mixed-list frequency effects in recall.
The crossover interaction of frequency and memory task has served as a benchmark finding for theoretical models of memory. The model of Grossberg and Stone (1986) and the retrieving effectively from memory (REM) model (Shiffrin \& Steyvers, 1997) are particularly relevant. In the REM model, features vary in their environmental frequency, and rare features are more diagnostic than frequent features. Representations of noncanonical viewpoints tend to be made up of less common features and, therefore, contain more diagnostic features for recognition memory than do the memory representations of canonical viewpoints. Within the framework of feature memory models, Malmberg and Nelson (2003) and Criss and Shiffrin (2004) have proposed that the word frequency effects in recognition memory might depend on which features are available during encoding, with early encoding benefiting low-frequency words. In our experiments, early encoding might have been directed toward surfacelevel features, where the unusual viewpoint could produce a distinctiveness advantage in recognition. Indeed, as can be seen in Figure 2 in the recognition memory panel, items studied in the noncanonical viewpoint tended to produce higher rates of "old" responses within each of the studytest conditions. How does this account explain the recallrecognition dissociation? Given the nature of the recall experiment, in which participants had to write down the names of the objects, participants might have focused instead on the semantic features, which might be more readily available for canonical viewpoints. This focus might have produced the advantage in Experiment 2.

In summary, objects depicted in the canonical and noncanonical viewpoints yielded differential effects in memory performance. These results provide some empirical support to the psychological reality of canonical viewpoints and to the frequency-based accounts of canonical viewpoint effects in object recognition. This dissociation is constraining for theories of viewpoint: If viewpoint functions like many other variables - such as study time or depth of processing - by affecting the strength of the memory trace, one would expect viewpoint to affect recognition and recall in the same direction. The rich set of results in the present study, and the range of manipulations that are possible with 3-D objects but not possible with word stimuli, might allow research on memory for 3-D objects to provide important insights about memory processes.

\section{AUTHOR NOTE}

This research was supported by National Science Foundation (NSF) Grant SES-0446869 to P.G., and NSF Grant SES-0351523 and NIMH Grant R01-MH071418 to J.N.R. Correspondence concerning this article should be addressed to P. Gomez, Department of Psychology, DePaul University, 2219 North Kenmore, Chicago, IL 60614 (e-mail: pgomez1@depaul.edu).

\section{REFERENCES}

BiEDERMAN, I. (1987). Recognition-by-components: A theory of human image understanding. Psychological Review, 94, 115-147.

Blanz, V., TARr, M. J., \& BÜlthoff, H. H. (1999). What object attributes determine canonical views? Perception, 28, 575-599.

CRISS, A. H., \& SHIFFrIN, R. M. (2004). Interactions between study task, study time, and the low-frequency hit rate advantage in recognition 
memory. Journal of Experimental Psychology: Learning, Memory, \& Cognition, 30, 778-786.

Edelman, S., \& Bülthoff, H. H. (1992). Orientation dependence in the recognition of familiar and novel views of three-dimensional objects. Vision Research, 32, 2385-2400.

Gillund, G., \& Shiffrin, R. M. (1984). A retrieval model for both recognition and recall. Psychological Review, 91, 1-67.

GlanZER, M., \& AdAMs, J. K. (1985). The mirror effect in recognition memory. Memory \& Cognition, 13, 8-20.

Glanzer, M., \& Bowles, N. (1976). Analysis of the word-frequency effect in recognition memory. Journal of Experimental Psychology: Human Learning \& Memory, 2, 21-31.

Grossberg, S., \& Stone, G. (1986). Neural dynamics of word recognition and recall: Attentional priming, learning, and resonance. Psychological Review, 93, 46-74.

HaLl, J. F. (1954). Learning as a function of word frequency. American Journal of Psychology, 67, 138-140.

Hockley, W. E. (1994). Reflections of the mirror effect for item and associative recognition. Memory \& Cognition, 22, 713-722.

JACOBY, L. L., \& DALlas, M. (1981). On the relationship between autobiographical memory and perceptual learning. Journal of Experimental Psychology: General, 110, 306-340.

JoLICEUR, P. (1985). The time to name disoriented natural objects. Memory \& Cognition, 13, 289-303.

KARLSEN, P. J., \& SNODGRASs, J. G. (2004). The word-frequency paradox for recall/recognition occurs for pictures. Psychological Research, 68, 271-276.

Karnath, H.-O., Ferber, S., \& Bülthoff, H. H. (2000). Neuronal representation of object recognition. Neuropsychologia, 38, 1235-1241.

Malmberg, K. J., \& Nelson, T. O. (2003). The word frequency effect for recognition memory and the elevated-attention hypothesis. Memory \& Cognition, 31, 35-43.

Palmer, S. E., Rosch, E., \& Chase, P. (1981). Canonical perspective and the perception of objects. In J. Long \& A. Baddeley (Eds.), Attention and performance IX (pp. 135-151). Hillsdale, NJ: Erlbaum.

Peters, H. N. (1936). The relationship between familiarity of words and their memory value. American Journal of Psychology, 48, 572-584.

Rouder, J. N., Ratcliff, R., \& McKoon, G. (2000). A neural network model of implicit memory in object recognition. Psychological Science, 11, 13-19.

Scarborough, D. L., Cortese, C., \& Scarborough, H. S. (1977). Frequency and repetition effects in lexical memory. Journal of Experimental Psychology: Human Perception \& Performance, 3, 1-17.

SHEPARD, R. N. (1967). Recognition memory for words, sentences, and pictures. Journal of Verbal Learning \& Verbal Behavior, 6, 156-163.

Shiffrin, R. M., \& Steyvers, M. (1997). A model for recognition memory: REM-retrieving effectively from memory. Psychonomic Bulletin \& Review, 4, 145-166.

TARR, M. J., \& BüLTHOFF, H. H. (1995). Is human object recognition better described by geon structural descriptions or by multiple views? Comment on Biederman and Gerhardstein (1993). Journal of Experimental Psychology: Human Perception \& Performance, 21, 1494-1505.

TARr, M. J., \& Pinker, S. (1989). Mental rotation and orientationdependence in shape recognition. Cognitive Psychology, 21, 233-282.

Underwood, B. J., Ekstrand, B. R., \& Keppel, G. (1965). An analysis of intralist similarity in verbal learning with experiments on conceptual similarity. Journal of Verbal Learning \& Verbal Behavior, 4, 447-462.

Underwood, B. J., \& Freund, J. S. (1970). Testing effects in the recognition of words. Journal of Verbal Learning \& Verbal Behavior, 9 , 117-125.

Verfaillie, K., \& Boutsen, L. (1995). A corpus of 714 full-color images of depth-rotated objects. Perception \& Psychophysics, 57, 925-961.

(Manuscript received November 1, 2007; revision accepted for publication April 7, 2008.) 